This item was submitted to Loughborough's Research Repository by the author.

Items in Figshare are protected by copyright, with all rights reserved, unless otherwise indicated.

\title{
Magnitude representations and counting skills in preschool children
}

PLEASE CITE THE PUBLISHED VERSION

http://dx.doi.org/10.1080/10986065.2015.1016811

\section{PUBLISHER}

(c) Taylor and Francis

\section{VERSION}

AM (Accepted Manuscript)

\section{PUBLISHER STATEMENT}

This work is made available according to the conditions of the Creative Commons Attribution-NonCommercialNoDerivatives 4.0 International (CC BY-NC-ND 4.0) licence. Full details of this licence are available at: https://creativecommons.org/licenses/by-nc-nd/4.0/

\section{LICENCE}

CC BY-NC-ND 4.0

\section{REPOSITORY RECORD}

Batchelor, Sophie, Sarah Keeble, and Camilla K. Gilmore. 2019. "Magnitude Representations and Counting Skills in Preschool Children". figshare. https://hdl.handle.net/2134/17015. 
Magnitude Representations and Counting Skills in Preschool Children Sophie Batchelor, Sarah Keeble and Camilla Gilmore Mathematics Education Centre, Loughborough University

\section{Author Note}

This research was supported in part by a British Academy Fellowship awarded to Camilla Gilmore.

Correspondence concerning this article should be addressed to Sophie Batchelor, Mathematics Education Centre, Loughborough University, Loughborough, Leicestershire, LE11 3TU, UK. E-mail: s.m.batchelor@lboro.ac.uk 


\begin{abstract}
When children learn to count, they map newly acquired symbolic representations of number onto preexisting nonsymbolic representations. The nature and timing of this mapping is currently unclear. Some researchers have suggested this mapping process helps children understand the cardinal principle of counting, while other evidence suggests that this mapping only occurs once children have cardinality understanding. One difficulty with the current literature is that studies have employed tasks that only indirectly assess children's nonsymbolic-symbolic mappings. We introduce a task in which preschoolers made magnitude comparisons across representation formats (e.g., dot arrays vs. verbal number), allowing a direct assessment of mapping. We gave this task to 60 children aged $2 ; 7-4 ; 10$, together with counting and Give-a-Number tasks. We found that some children could map between nonsymbolic quantities and the number words they understood the cardinal meaning of, even if they had yet to grasp the general cardinality principle of counting.
\end{abstract}

Keywords: counting, magnitude comparison, cardinality, preschool children, number 


\section{Magnitude Representations and Counting Skills in Preschool Children}

We know from more than a decade's worth of research that infants, children and adults can represent and manipulate numerical information nonsymbolically, without number words or digits. These nonsymbolic representations are robust across multiple modalities and set sizes. Children and adults can compare, add and subtract small and large quantities in visual arrays (Barth, Kanwisher, \& Spelke, 2003; McCrink \& Wynn, 2004), auditory sequences (Barth et al., 2003; Barth, La Mont, Lipton, \& Spelke, 2005) and moving displays of actions (e.g., puppet jumps) (Wood \& Spelke, 2005; Wynn, 1996; Wynn, Bloom, \& Chiang, 2002).

The nonsymbolic representations employed in these tasks are approximate and in an analogue format. They are inherently noisy and the variance associated with them increases with the absolute size of the magnitudes represented. As a result, success on these tasks depends on the ratio (or numerical distance) between the numerosities to be compared ${ }^{1}$. As the quantities get closer together, discrimination becomes more effortful and less precise. Importantly, the precision of these representations varies across individuals and increases over development. Infants can discriminate numerosities with ratios as small as 2:3, whilst preschool children show a ratio-limit of 3:4, and adults, 7:8 (Barth et al., 2003; Feigenson, Dehaene, \& Spelke, 2004).

When children begin to count they learn to use external symbols to represent number. These symbolic representations enable exact number comparison and manipulation. There is evidence that when children acquire this symbolic system, the preexisting nonsymbolic system is not overridden; rather, nonsymbolic representations become mapped onto the newly acquired symbolic representations. The evidence for this is at least threefold. Firstly, children and adults show a numerical distance effect for symbolic number comparison (Dehaene, Dupoux, \& Mehler, 1990; Moyer \& Landauer, 1967; Temple \& Posner, 1998). When asked to compare two symbolic numbers (e.g., Arabic digits) reaction times and 
accuracy vary systematically with the numerical distance between the symbols. As with nonsymbolic comparison, performance is slower and less accurate as the numbers to be compared get closer. This suggests that symbolic numbers are mapped onto, and automatically activate, approximate nonsymbolic representations. Secondly, evidence for these mappings come from investigations showing that children and adults can perform rapid approximate arithmetic on symbolic numbers (e.g., Gilmore, McCarthy, \& Spelke, 2007; Xenidou-Dervou, De Smedt, van der Schoot, \& van Lieshout, 2013). These approximate computations engage a distinct neural system to that which is activated during exact symbolic arithmetic (Dehaene, Spelke, Pinel, Stanescu, \& Tsivkin, 1999). Thirdly, some neuropsychological studies have revealed associations between the nonsymbolic and symbolic systems (Dehaene, Dehaene-Lambertz, \& Cohen, 1998). Impairments in nonsymbolic processing are associated with impairments in the symbolic domain, and vice versa.

This evidence suggests that, in individuals who have already acquired the symbolic system, symbolic representations are connected with preexisting nonsymbolic representations. However, we do not know how these symbolic-nonsymbolic mappings are formed, or whether they play a role in the acquisition of symbolic mathematical skills. Below we review evidence concerning the relationship between nonsymbolic and symbolic representations and mathematics, before turning to evidence concerning how these mappings are formed.

\section{Magnitude Representations and Mathematics}

Children and adults show individual differences in the precision of their numerical magnitude representations (Barth et al., 2003; Feigenson et al., 2004). The precision of these representations is typically indexed by performance on magnitude comparison tasks, either nonsymbolic (dot comparison) or symbolic (Arabic digit comparison). Participants are 
presented with two numerosities (dot arrays or Arabic digits) and are asked to select the more numerous. Several researchers have explored the relationship between children's and adults' performance on these tasks with performance on standardised tests of mathematics achievement. The evidence for a relationship between mathematics and nonsymbolic comparison performance has been somewhat mixed (see meta-analyses by Chen \& Li, 2014; Fazio, Bailey, Thompson, \& Siegler, 2014), although there is more consistent evidence for a relationship between mathematics and symbolic comparison performance (De Smedt, Noël, Gilmore, \& Ansari, 2013).

These findings have been taken to suggest that the mapping between nonsymbolic and symbolic representations may play a more pivotal role in later mathematics achievement than nonsymbolic acuity itself. It is not clear, however, whether the symbolic comparison task is measuring the mapping between nonsymbolic and symbolic representations, or whether it is measuring the nature of nonsymbolic or symbolic skills per se. To understand the nature of the mapping between representations and importantly, how this mapping is related to formal mathematics, we need more direct measures of mapping ability. Mundy and Gilmore (2009) introduced a novel mapping task in which children had to decide which of two nonsymbolic arrays matched a symbolic numerosity (symbolic to nonsymbolic mapping) or which of two symbolic numerosities matched a nonsymbolic array (nonsymbolic to symbolic mapping). Here it was found that individual differences in mapping ability accounted for variation in school mathematics achievement over and above standard symbolic and nonsymbolic numerical comparison tasks. Similarly Brankaer, Ghesquière, and De Smedt (2014) found that children's performance on a similar mapping task developed from age 6 to 8 and was related to performance on both timed and untimed mathematics tests, after controlling for magnitude comparison performance. These findings concur with data from Booth and Siegler (2008), which showed a relationship between children's ability to map symbolic 
representations onto a number line and later arithmetical achievement. These findings raise the question whether nonsymbolic representations and the mapping between nonsymbolic and symbolic representations play an instrumental role in the acquisition of symbolic representations. We turn to evidence on this point in the next section.

\section{Magnitude Representations and Learning to Count}

Several researchers have explored whether the formation of mappings between symbolic and nonsymbolic representations are critical for the acquisition of symbolic number knowledge (e.g., Le Corre \& Carey, 2007; Wagner \& Johnson, 2011). One possibility is that when children learn to count, they map newly acquired symbolic representations onto their preexisting nonsymbolic representations, and that the nonsymbolic system and in particular this mapping process helps with the acquisition of numbers (e.g., Gallistel \& Gelman, 1992). Alternatively, the symbolic system may be acquired independently of the nonsymbolic system and the mapping between nonsymbolic and symbolic representations might occur later, once children have acquired the symbolic system (e.g., Le Corre \& Carey, 2007). There is currently evidence for both of these accounts.

Before we review this evidence, we briefly describe three tasks typically used to measure children's counting skills and knowledge. Count list elicitation (or 'How high?') tasks are used to assess children's ability to generate the words in the count list. In some versions of this task children are simply asked to count up as high as they can and are scored based on the highest number they recall before making any errors (e.g., Barth, Starr, \& Sullivan, 2009; Lipton \& Spelke, 2005). In other versions of the task children are provided with a set of objects and are asked to count them (e.g., Le Corre \& Carey, 2007; Slaughter, Kamppi, \& Paynter, 2006). Meanwhile, 'How many?' and 'Give-a-Number' tasks are used to assess whether children understand the meaning of the words in the count list. They are assumed to measure children's knowledge of cardinality; i.e. understanding that the last word in their 
count list represents the numerosity of the set as a whole (Gelman \& Gallistel, 1978). In the 'How many?' task children are presented with a set of objects and they are asked to tell the researcher how many there are (unlike a count elicitation task with objects, children are not given the instruction to count the objects). In the 'Give-a-Number' task children are asked to give the researcher a requested number of objects. In both of these tasks children receive a score based on the highest number they can reliably produce.

A number of studies have explored the relationship between children's ability to compare nonsymbolic representations and their knowledge of the symbolic number system. HuntleyFenner and Cannon (2000) gave children aged 3-5 years a nonsymbolic (dot) comparison task and two to four weeks later they assessed verbal counting ability. Counting was assessed with a 'How high?' task and a 'How many?' task involving sets of 1-15 cubes. In line with previous studies, children's performance on the dot comparison task varied as a function of numerical ratio, thus demonstrating the signature ratio-effect of the nonsymbolic system. Correlation analyses revealed that the size of this ratio-effect was related to children's performance on the 'How high?' task, but not the 'How many?' task. Children with smaller ratio-effects, and therefore more precise nonsymbolic representations, were better able to recite the number words in their count list; however, they were no better able to understand the meanings of these words. Further to this, data from Slaughter et al., (2006) showed that preschool children's performance on a nonsymbolic subtraction task was unrelated to their ability to enumerate sets of up to 30 stickers. Together, these findings were taken to suggest that nonsymbolic magnitude representations develop independently of children's number word mappings and therefore the nonsymbolic system does not play a role in helping children acquire symbolic number.

More recent research has questioned this conclusion. Wagner and Johnson (2011) highlighted some methodological limitations of the counting tasks used in these studies. 
Specifically, they emphasised that children's success on a 'How many?' task does not necessarily reflect an understanding of the counting system. Children may learn to report the final tag of a count in response to 'How many?' without any conceptual understanding of cardinality. Indeed, previous research has shown that the 'How many?' task is a less reliable measure of whether children understand the counting system than a 'Give-a-Number' task in which children are asked to produce sets of items (Wynn, 1992). In view of this, Wagner and Johnson provided a further test of the relationship between children's nonsymbolic skills and their cardinality understanding using a variation of the 'Give-a-Number' task. In contrast to previous studies, results revealed a positive correlation between children's performance on the nonsymbolic comparison task and their cardinality understanding. Mussolin, Nys, Leybaert, and Content (2012) also found an association between children's nonsymbolic acuity and performance on a 'Give-a-Number' task. Interestingly, this correlation was significant for the younger children, aged 3-4 years, but not the older children, aged 5-6 years. This suggests that the role of nonsymbolic representations may change over development and highlights the importance of testing these abilities in children who are at the start of learning counting skills.

The studies by Wagner and Johnson (2011) and Mussolin et al. (2012) suggest that the precision of the nonsymbolic system is related to children's counting ability and therefore the nonsymbolic system may be involved in the acquisition of the symbolic number system. To measure nonsymbolic skills, these studies have used dot comparison tasks. Despite being widely used, dot comparison tasks have yet to be standardised making it difficult to compare the results across studies. One issue is that studies have employed different controls over the nonnumerical aspects of the dot stimuli. Some studies control for dot size and envelope area whilst others control for only one (or none) of these factors. Wagner and Johnson (2011), for example, only controlled for area. They note that children may have solved the task by 
simply adopting a strategy of selecting the array with the smaller dots. This makes it difficult to draw conclusions from their study. More recent research has suggested that inhibitory control processes can affect an individual's dot comparison performance (Gilmore et al., 2013). Therefore, any relationship between nonsymbolic comparison performance and symbolic number skills may be an artefact of nonnumerical task features. Moreover, to allow sufficient control for visual features of the displays, dot comparison tasks typically require a large number of trials to provide a reliable estimate of nonsymbolic acuity (e.g., Inglis \& Gilmore, 2014, suggest a minimum of 80 trials). This makes the dot comparison task unsuitable for use with young children.

Turning to studies that have employed tasks to measure symbolic-nonsymbolic mappings, rather than measures of the nonsymbolic system itself, there remain mixed findings. Lipton and Spelke (2005) studied preschool children's mapping of large number words to nonsymbolic numerosities. They gave children a counting assessment together with three tasks designed to measure knowledge of number word mappings: 1) An estimation ('How many on this card?') task, 2) a number word comprehension task and 3) a number word ordering task. Results showed that children could only map number words for those numbers within their counting proficiency level. Children who failed to count beyond 60 failed estimation, comprehension and ordering tasks for number words larger than 60 . The authors conclude that children map nonsymbolic and symbolic number representations at around the time that they master the count sequence. However, the children in this study were aged between 5 years and 6 years 2 months and are likely to have had some years of experience with counting.

In a follow-up study, Barth et al., (2009) examined the mappings of children with less counting knowledge. Here they found that whilst the least skilled counters did not produce accurate estimates on an estimation task, they did produce significantly larger estimates for 
larger sets. This suggests that even very inexperienced counters have some knowledge of large number word mappings. Similarly, Wagner and Johnson (2011) found that preschool children showed scalar variability in their mappings of number words that were beyond their cardinality proficiency. Together, these results suggest that children start to map nonsymbolic representations onto newly acquired symbolic representations before they have mastered the count sequence.

In contrast, other research suggests that number word mappings develop after children understand the number system. Le Corre and Carey (2007) gave children aged 3-5 years a count list elicitation task, a 'Give-a-Number' task, an estimation task and a nonsymbolic ordinal judgment task. They classified children into number knower-levels based on the highest number they reliably produced on the 'Give-a-Number' task. Children were either classified as subset-knowers (one-knowers, two-knowers, three-knowers or four-knowers) or cardinal principle-knowers (CP-knowers). Le Corre and Carey found that none of the subsetknowers and only half of the CP-knowers could map beyond four on the estimation task without counting. They argue that the presence of a group of CP nonmappers suggests that the mapping of number words beyond four is not part of the acquisition of the counting principles. They further specify that number words beyond four are mapped onto nonsymbolic numerosities about six months after children acquire the counting principles.

Data from Slusser and Sarnecka (2011) provides additional support for this view. Children aged 2-4 years completed a 'Give-a-Number' task and a word extension task with two alternative forced-choice options. The results revealed that only CP-knowers were above chance at extending number words ("four", "five", "eight" and "ten") from one set to another based on numerosity. In contrast, all children succeeded at matching pictures by mood or colour. In a follow-up experiment, Slusser and Sarnecka found that even CP-knowers had difficulty extending words by number on trials where mood and colour were incongruent. 
These results further suggest that children do not map number words to nonsymbolic representations until they understand the cardinal principle of counting.

Summarising these studies, there is inconsistent evidence regarding the relationship between young children's nonsymbolic skills and their knowledge of the counting system. Specifically, we do not yet know whether young children can map number words to nonsymbolic representations of quantity before, or only after they grasp the cardinality principle of counting.

\section{Methodological Issues}

One reason for the inconsistent findings in the literature is that studies have used different tasks to measure counting skills. These tasks tap into different aspects of children's counting knowledge - from number word sequence production to cardinality understanding and they vary in terms of their reliability. Specifically, it is not clear whether performance on the 'How many?' task reflects any true conceptual understanding of cardinality. As noted previously, it is thought to provide a weaker measure of cardinality than the 'Give-a-Number' task.

To assess the mapping between nonsymbolic-symbolic representations, researchers have used symbolic comparison tasks, matching tasks or estimation tasks. The symbolic comparison task is limited because it only provides an indirect measure of mapping skills. Estimation tasks provide a more direct measure of mapping; however, they are also limited due to the task demands placed on participants. Typically in these tasks participants are presented with arrays of nonsymbolic (dot) stimuli and are asked to estimate the number of dots. Studies have shown that, unless calibrated, adults' estimates are generally inaccurate (e.g., Izard \& Dehaene, 2008) and many children fail to produce estimates at all (e.g., Lipton $\&$ Spelke, 2005). The use of these free-response tasks with preschool children is therefore questionable. Mundy and Gilmore (2009) developed a mapping task for use with school age 
children which allows a more direct assessment of children's mapping between symbolic and nonsymbolic quantities. However, this still requires mapping skills to be assessed in both directions (i.e. symbolic to nonsymbolic and nonsymbolic to symbolic) which increases the number of trials, making it less suitable for use with young children. In view of these difficulties, here we introduce a cross-notation comparison task in which preschool children made magnitude comparison judgements across representational formats (e.g., dot arrays vs. verbal number words). This allowed a direct assessment of the mapping between magnitude representations that is suitable for use with young children.

Finally, a number of previous studies have been conducted with children who may have had several months or years experience with counting. These studies cannot reveal the processes involved in the very early stages of number acquisition. To determine whether magnitude representations are involved in the process of learning to count, it is essential to study these effects in children who are not yet experienced counters.

\section{The Present Study}

In this study we used a novel mapping task to investigate the link between preschool children's magnitude representations and their early counting skills. We gave children aged 2-4 years three tasks designed to measure separate competencies. Firstly, a count list elicitation task was used to assess children's mastery of the verbal count list. Secondly, a 'Give-a-Number' task was used to assess children's cardinality understanding. Finally, a cross-notation comparison task was used to assess children's mapping between nonsymbolic and symbolic magnitude representations. If, as found by Lipton and Spelke, (2005), children's ability to map number words to nonsymbolic numerosities is limited by knowledge of the count list then we would expect performance on the count list elicitation task to be the best predictor of performance on the cross-notation comparison task (Prediction 1). Alternatively, performance on the cross-notation comparison task may be limited by 
knowledge of cardinality. In this case we would expect only children who are CP-knowers on the Give-a-Number task to be able to complete the cross-notation comparison task (Prediction 2). Finally, if children map number words to quantities during the process of acquisition and before they understand the cardinality principle then we would expect some children who are not CP-knowers to be able to complete the cross-notation comparison task (Prediction 3). If there is no evidence that children can complete the cross-notation comparison task before they are $\mathrm{CP}$ - knowers then this suggests that symbolic-nonsymbolic mappings do not play a critical role in acquiring the count sequence and thus these mappings must be formed later, once children are proficient with the symbolic number system. Alternatively, if children are able to map between symbolic and nonsymbolic representations before they are $\mathrm{CP}$ knowers then this suggests that these mappings must form while children are learning the number symbols, and may play a critical role in this process.

\section{Method}

\section{Participants}

Sixty preschool children (31 male) aged between 2 years 7 months and 4 years 10 months (mean age 3 years 8 months) participated in the study. Children were recruited either through local nurseries in Nottingham, UK, or through the University of Nottingham's Human Development and Learning participant database. Testing took place either at a child's nursery or at a university laboratory. Participation was voluntary and all children received stickers for taking part. Children invited into the university also received a small gift to thank them for taking part and caregivers received reimbursement for their travel expenses.

Seven children were excluded from all the analyses for the following reasons: English was not their native language $(n=2)$, failure to attempt all tasks $(n=4)$, unable to produce an accurate count list $(n=1)$. A further seven children attempted all tasks but completed 
insufficient trials of the comparison task to allow analysis. These participants were excluded from the analyses that involved the comparison task. Thus there were 46 complete datasets.

\section{Tasks}

Each child was presented with three tasks: a count list elicitation task (based on Le Corre \& Carey, 2007), a Give-a-Number task (based on Wynn 1990, 1992) and a novel crossnotation magnitude comparison task. These tasks allowed us to assess mastery of the verbal count list, cardinality understanding and mapping between magnitude representations (nonsymbolic and symbolic), respectively.

Count list elicitation task. The experimenter placed 20 small objects in a single row in front of the child and asked them to count them aloud. The objects were identical in all respects. Children were given a choice as to which character they wished to play the game with and this character choice determined the set of objects used (felt strawberries, felt bananas, plastic bricks).

If the child refused to produce a count list or if they produced a count list without attending to the objects, the experimenter prompted them: "Can you count them with me...one, two...," whilst pointing at the objects. Each child was assigned a counting score based on the highest number that they counted to correctly, regardless of whether they pointed to the objects whilst doing so.

Give-a-Number task. Adapted from Wynn $(1990,1992)$ this task was presented in line with previous studies (e.g., Condry \& Spelke, 2008; Le Corre \& Carey, 2007; Sarnecka \& Gelman, 2004). The experimenter placed 15 objects in a random cluster in front of the child. The child was then asked to give their chosen character [character $\mathrm{x}$ ] a given number [n] of the objects. For example, "Can you give Mike the Monkey three bananas?" Following the child's response, the experimenter asked; "Is that [n]?" If the child responded with "yes", the 
experimenter proceeded with the next trial. If the child responded with "no", the experimenter repeated the original request.

The numbers 1-6 were requested in a pseudorandom order. The experimenter began by asking the child to give 3 objects, and then increased or decreased the number accordingly. Each number was requested up to three times; if a child succeeded once and failed once at a given number, it was asked for a third time. Incorrect trials on which a child produced a different number in the experimental range (1-6) were marked as incorrect for both the requested and the produced number.

Children were categorised as knowers of a given number when they succeeded on two of the three trials for that number (whilst also producing that number no more than half as often when asked for a different number). Each child was assigned a number knower-level based on the highest number that they reliably produced. For example, children who reliably produced sets of 1 and 2 and 3 objects, but not 4 objects, were categorised as a threeknowers. Children who succeeded at the highest sets (5 and 6) were categorised as cardinal principle-knowers (CP-knowers).

Cross-notation comparison task. In 12 experimental trials, children were instructed to choose which of two characters had the most balls. The experimenter presented two numerosities sequentially. The first numerosity was presented nonsymbolically as an array of dots on a card $(15.5 \mathrm{~cm} \times 10.5 \mathrm{~cm})$. As the experimenter presented the card they said: "[Character $\mathrm{x}]$ has this many balls." The second numerosity was presented symbolically as a verbal number word, read aloud by the experimenter. To aid conceptualisation, the experimenter presented a picture of a box on a card (the same size as the dot array cards) and said: "[Character $\mathrm{x}]$ has hidden his/her balls in a box. [Character $\mathrm{x}]$ has [n] balls." The experimenter then asked: "Who has the most balls?" Both stimulus cards remained in place next to each of the characters until the child gave their response (see Figure 1b). Children 
responded by naming and/or pointing to the character. Children were instructed not to use counting to solve this task and if the experimenter saw children begin to make any visible counting acts, they were reminded not to.

The numerosities used ranged between 1-10. One third of the trials involved at least one numerosity within the subitising range (1-3) and two thirds of the trials involved two numerosities outside the subitising range (4-10). The numerical distance between the quantities being compared was varied; half of the trials had a small numerical distance (a distance of 1,2 or 3) and half of the trials had a large numerical distance (a distance of 4 or 5). Each type of stimulus (dot array/verbal number word) was the larger amount an equal number of times, and each character had the larger amount an equal number of times (see Table 1). Dot arrays were generated randomly. All of the dots on a given stimulus card were identical, however, colour (blue, green, red) and dot size (small, medium, large) were varied across stimulus cards.

The experimenter presented Trials 1 and 2 successively, followed by Trials 3-12 in a random order for each child. Trials 1 and 2 were presented first because they involved small numbers (within the subitising range) only. Prior to the experimental trials, children completed two nonsymbolic practice trials (dot array vs. dot array) to familiarise them with the rules of the game. In Practice Trials 1 and 2 children were asked to compare 2 dots vs. 1 dot and 4 dots vs. 2 dots (see Figure 1a).

\section{Procedure}

Children were tested individually, in a single 10-20 minute session, either at their nursery or a university laboratory. Children tested at nursery were accompanied by a member of the nursery staff and children tested at the university were accompanied by their caregiver.

The experimenter sat opposite the child and presented all three tasks sequentially: 1) Count list elicitation, 2) Give-a-Number and 3) Cross-notation comparison. The children 
received a sticker after each task. The experimenter emphasised that they were number games and children were encouraged to have a guess even if they were not sure. Throughout the experiment, feedback on accuracy was not given; however, children received praise for their general attention and behaviour.

\section{Results}

In presenting the results, we first provide a descriptive overview of the children's performance on each of the three tasks. In the sections that follow, we test the alternative predictions derived from the previous literature. First we use correlations and regression to explore the relationships among performance on each task and test whether count list knowledge (Prediction 1) or cardinality knowledge (Predictions 2 and 3) was a stronger predictor of performance on the cross-notation comparison task. In order to further tease out the importance of full cardinal knowledge (Prediction 2) versus an immature cardinal knowledge of a subset of numbers (Prediction 3) on performance on the cross-notation comparison task, we consider performance on the cross-notation comparison task for groups of children at different knower-levels in detail. Finally, we explore individual patterns of performance across the cross-notation comparison and Give-a-Number tasks as a further test of Predictions 2 and 3.

Children showed a range of performance on all of the tasks. The highest number reached in the counting task ranged from 8 to 20 with a mean of $14.7(S D=3.9)$. Mean knower-level on the Give-a-Number task was $3.85(S D=2.2$, range $0-6)$. Mean accuracy on the crossnotation comparison task was $0.72(S D=0.2$, range $0.3-1)$. This indicates that these tasks were appropriate for use with this group of preschool children. Cronbach's alpha for the Give-a-Number task was 0.76 and for the cross-notation comparison task was 0.64 . We explored performance on the cross-notation comparison task to consider whether there was any evidence that children were using covert counting to solve this task. Using a by-items 
analysis we found that accuracy was not related to the size of the dot array $(r=-.042, p=$ $.897)$ but was strongly related to the size of the verbal number word $(r=-.860, p<.001)$. These correlations were significantly different $(t(9)=3.74, p=.005)$. This suggests that children were not using counting to solve this task, since young children's counting performance is related to set-size (Fuson, Pergament, Lyons, \& Hall, 1985).

Table 2 reports the number of children achieving each knower-level on the Give-aNumber task. The distribution of knower-levels and ages is in line with previous research (Le Corre \& Carey, 2007). The absence of five-knowers in our sample suggests that once children become four-knowers, they quickly move on to becoming cardinal principleknowers. The ages of our participants ranged from 2 years 7 months to 4 years 10 months, a period that is rich in developmental change. Therefore we looked at whether children's age in months was related to their performance on each task. Age at testing was significantly related to performance on all three tasks (Give-a-Number $r=.80, p<.001$; cross-notation comparison $r=.64, p<.001$; count list elicitation $r=.72, p<.001)$; thus we included age as a covariate in our further analyses.

\section{Correlations Among Tasks}

We performed a series of correlations in order to explore relationships among the tasks. There were positive correlations among children's performance on each of the three tasks (see Figure 2). Give-a-Number task knower-level was correlated with accuracy on the crossnotation comparison task, $r=.71, p<.001$ (controlling for age $r=.41, p=.005$ ) and with the highest number reached in the count list elicitation task $r=.70, p<.001$ (controlling for age $r$ $=.32, p=.022$ ). Accuracy on the cross-notation task was also correlated with the highest number reached in the count list elicitation task $r=.58, p<.001$ (controlling for age $r=.268$, $p=.079)$. These relationships were then subjected to partial correlations in order to explore whether they represented independent relationships. Knower-level was still correlated with 
accuracy on the cross-notation comparison task after controlling for count list knowledge $r=$ $.53, p<.001$ and knower-level was correlated with the highest number reached in the count list elicitation task after controlling for accuracy on the cross-notation comparison task $r=$ $.44, p=.003$. However, accuracy on the cross-notation comparison task was no longer correlated with the highest number reached on the count list elicitation task after controlling for knower-level $r=.22, p=.16$. In keeping with this, in a linear regression predicting performance on the cross-notation comparison task $\left(r^{2}=.53, p<.001\right)$, knower-level was found to be a significant independent predictor $(\beta=.45, t=2.50, p=.016)$ but count list knowledge $(\beta=.17, t=1.09, p=.282)$ and age at testing $(\beta=.18, t=0.95, p=.346)$ were not.

This analysis indicates that knower-level is a better predictor of cross-notation comparison performance than count list knowledge or age, providing evidence against Prediction 1 and in support of Prediction 2. Therefore, it is understanding of the cardinal principle, rather than simply knowledge of the count sequence, which limits children's ability to map number words to nonsymbolic quantities.

\section{Cross-notation Comparison Task Performance of Children at Different Knower-levels}

If cardinal principle knowledge limits performance on the cross-notation comparison task, is full cardinal principle understanding required for the task, in line with Prediction 2, or is an immature cardinal understanding of only a subset of numbers sufficient, as suggested in Prediction 3? We explored performance on the cross-notation comparison task by children at different knower-levels, using a one-way $\mathrm{ANOVA}^{2}$ (see Figure 3). The participants were grouped into one of three knower-level groups: one/two-knowers, three/four-knowers, and CP-knowers. There was a significant effect of knower-level on cross-notation comparison task accuracy, reflecting the fact that children who were at a higher knower-level were more accurate, $F(2,42)=25.0, p<.001$. Bonferroni-corrected t-tests indicated that the mean 
scores for the one/two-knowers $(\mathrm{M}=.54)$ and the three/four-knowers $(M=.68)$ did not differ significantly $(p=.065)$, but the CP-knowers $(M=.86)$ were significantly more accurate than the one/two-knowers $(p<.001)$ and the three/four-knowers $(p=.008)$.

The cross-notation comparison task requires a choice to be made from two options for each trial, and therefore chance performance was $50 \%$. We explored whether children in each of the groups performed above chance on the task using one-sample t-tests. CP-knowers and three/four-knowers both performed above chance $(t(22)=11.93, p<.001$ and $t(7)=3.41, p=$ .011 respectively). However, one/two-knowers were not significantly above chance, $t(13)=$ $1.28, p=.224$

These results suggest that children are able to map numerical symbols onto nonsymbolic quantities once they have understood the cardinal meaning of some numbers (i.e. are at least three/four-knowers), but the accuracy of the connection between numerical symbols and nonsymbolic quantities improves once children are cardinal principle-knowers. This appears to give a mixed picture regarding the relationship between these skills; children who are not yet CP-knowers could perform the cross-notation comparison task to some extent, but their performance was lower than the children who were CP-knowers, which does not provide specific support for either Prediction 2 or 3.

However, in these analyses we considered all trials of the cross-notation comparison task. For the children who were not yet CP-knowers this included some trials that were within their knower-level, but also some trials which were within their count list but above their knowerlevel (e.g., asking a three-knower to compare six dots versus the number word "nine"). In order to determine whether children who were not CP-knowers were able to connect number words and nonsymbolic quantities within their knower-level range, we calculated a new set of accuracy scores for those trials which contained only verbal numbers falling within the knower-level of each participant. For example, for a participant who was a three-knower the 
new accuracy score only included trials where the verbal number word ranged from one to three. Therefore performance was considered across a different number of trials for children at different knower-levels (12 trials for CP-knowers, 5 trials for four-knowers, 3 trials for three-knowers and 2 trials for two-knowers). Note that there were no trials containing the verbal number word "one" therefore one-knowers were excluded from the following analyses.

We re-ran the ANOVA 2 examining the effect of knower-level on performance on the cross-notation comparison task. This time there was no effect of knower-level on accuracy, $F(2,37)=.67, p=.519$ (see Figure 3$)$. We also used these new accuracy scores to compare performance to chance level for both of the non-CP-knower groups. This time the three/fourknowers were found to be performing significantly above chance, $t(7)=3.12, p=.017$, and performance of the two-knowers approached significance $t(8)=2.29, p=.051$.

These results suggest that, rather than only CP-knowers being able to complete the crossnotation comparison task in line with Prediction 2, children who are not yet CP-knowers are accurate at mapping between nonsymbolic and symbolic representations when the verbal number falls within their knower-level range. Thus there is evidence that children who do not understand the cardinality principle are able to map number words that they understand the cardinal meaning of to nonsymbolic representations, providing some support for Prediction 3.

If it is the case that both CP- and non-CP-knowers can map some verbal number words onto nonsymbolic quantities, then, in line with theories of magnitude representations (Feigenson et al., 2004), we would expect both groups to show a numerical distance effect in their responses to the cross-notation comparison task. We therefore conducted a two-way mixed design ANOVA with numerical distance (small, large) and knower-level (CP-knower, non-CP-knower) as factors. Due to the small number of trials which fall within their knowerlevel, the one/two-knowers were not included in this analysis. As expected, there was a 
significant effect of distance $(F(1,29)=6.955, p=.013)$ with more accurate performance for trials with a large $(M=0.85)$ compared to a small $(M=0.77)$ numerical distance, a signature of the nonsymbolic system. Importantly, there was no significant interaction between group and distance $(F(1,29)<1, p=.510)$, indicating that both groups showed an equivalent distance effect. The existence of the numerical distance effect in this task reflects the approximate nature of the nonsymbolic system, providing evidence that the cross-notation comparison task measures the ability to map between the two systems in both CP-knowers and non-CP-knowers.

\section{Individual Patterns of Performance}

Finally, to further distinguish between Predictions 2 and 3, we explored individual patterns of performance across the Give-a-Number and cross-notation comparison tasks. The group-level analyses reported above suggest that, at a group-level, children were able to complete the cross-notation comparison task within their knower-level even if they were not CP-knowers on the Give-a-Number task. However, analysis of individual patterns of performance can reveal how frequent this pattern of performance was. We therefore scored the tasks on a pass/fail basis, scoring ' 1 ' if they passed, and ' 0 ' if they did not. Children were considered to have passed the Give-a-Number task, if they were CP-knowers and were considered to have passed the cross-notation comparison task if they scored above chance level according to a binomial distribution (in the case of two-knowers if they answered all items correctly). In line with the analysis above, we considered only the trials that fell within an individual's knower-level. We found that 9 children (21\%) failed both the comparison task and the Give-a-Number task and 16 children (38\%) passed both tasks. Of the remaining children, 8 (19\%) passed the Give-a-Number task (i.e. were CP-knowers) but failed the comparison task and $9(21 \%)$ passed the comparison task but failed the Give-a-Number task (i.e. were not CP-knowers). It is clear that there is no consistent order in which children were 
able to pass the Give-a-Number and cross-notation comparison tasks, lending mixed support for both Predictions 2 and 3.

\section{Discussion}

In this study we explored how children's mappings between symbolic and non-symbolic numerical representations are formed and whether they are involved in the acquisition of symbolic skills. We found evidence that some children can map between nonsymbolic representations and number words (within their knower level) before they are cardinal principle knowers. This suggests that, for some children at least, these mappings form while the symbolic system is being acquired, and may therefore play a role in this process.

Our results reveal that there is a complex relationship between count list knowledge, understanding of the cardinality principle and the ability to map between number words and nonsymbolic quantities. These findings have both theoretical and methodological implications for the debate surrounding the role of nonsymbolic representations and mappings between nonsymbolic and symbolic representations in the acquisition of symbolic representations. Below we first review the theoretical conclusions that can be drawn from this work before considering the methodological contributions. We end by considering the implications for supporting the development of young children's numeracy skills.

\section{Theoretical Implications Regarding the Development of Symbolic-Nonsymbolic}

\section{Mappings}

Contrary to Prediction 1, our results clearly demonstrate that it was knowledge of cardinality, rather than count list knowledge, which was an important determinant of children's ability to map between symbolic and nonsymbolic representations. Cardinality knowledge was related to mapping skill even after controlling for age. Previous studies have shown that children may be able to accurately recite a count list some time before they grasp the concept of cardinality (e.g., Wynn, 1990). We add to these findings by showing that count 
list knowledge is also not sufficient for children to be able to map across symbolic and nonsymbolic representations. Although children may learn the sequence of number words at an early age, it is only when they have both grasped the concept of cardinality and mapped these number words onto representations of magnitude that they can be considered to have understood the meaning of their count list.

In contrast to Le Corre and Carey (2007) and Slusser and Sarnecka (2011) we found that children could map between number words within their knower-level and nonsymbolic quantities even if they did not grasp the general cardinality principle, in line with Prediction 3. Both group-level and individual-level analyses revealed that being a cardinal principleknower was not essential in order for children to correctly compare number words and quantities that were within their knower-level. However, it does not appear that all children necessarily develop these skills in a consistent order lending partial support to both Predictions 2 and 3. Our findings in relation to Prediction 3 are tentative given that this was based on the analysis of only a small number of trials. Future research should seek to replicate this with studies in which the trials of the cross-notation comparison task are carefully selected in light of children's knower-level.

One possible explanation of the differences in our findings compared to the previous literature might lie in the nature of the mapping task employed. The tasks used by Le Corre and Carey (2007) required children to produce a verbal estimate for a nonsymbolic array. As highlighted by Lipton and Spelke (2005), young children may be reluctant to make guesses in a free estimate situation. Therefore, this task may have underestimated children's knowledge. Our cross-notation comparison task did not require free estimate production and thus the general demands of the task were lower. In this way we were able to reveal children's mappings between number words and nonsymbolic quantities at an earlier age. 
The inconsistent patterns revealed by our individual-level analyses can be interpreted in two alternative ways. First, it may be that cardinality understanding and the ability to map between number words and quantities develop together but are initially fragile skills. As children first acquire these skills there may be a period of time in which they are not able to demonstrate this ability reliably in all contexts. A similar pattern is observed in the development of children's understanding of commutativity whereby task features affect children's early ability to demonstrate understanding of commutativity (Cowan, Dowker, Christakis, \& Bailey, 1996). Thus, for example, a child may show evidence of cardinality understanding in one situation but not another. Over time this understanding becomes more reliable across all contexts (c.f. Baroody, Wilkins, \& Tiilikainen, 2003). Our pattern of apparently inconsistent individual-level results may have arisen because some children were unable to reliably reveal their understanding of cardinality or their ability to map between number words and quantities.

On the other hand, it is possible that these findings reveal that there are alternative developmental pathways in which children can acquire understanding of cardinality and the ability to map number words to quantities. Some evidence suggests that there may be individual differences in development pathways for other arithmetic concepts (e.g., Gilmore \& Papadatou-Pastou, 2009). For some children, knowledge of the symbolic number system may take priority and they acquire understanding of the cardinality principle before they make the connection between this system and the nonsymbolic system of magnitude representations. For other children, the connection between the systems may take priority and mappings develop at an earlier stage. Up until now, the existence of alternative pathways for the development of early symbolic skills has yet to be considered (Le Corre \& Carey, 2007; Wagner \& Johnson, 2011). 
In order to distinguish between these possibilities, longitudinal research is needed in which individual children's performance is considered as they develop these two skills. Given the rapid pace in which changes to early number understanding occur, detailed microgenetic methods (Siegler \& Crowley, 1991) would be appropriate to capture subtle changes in children's understanding. This would reveal whether all children follow the same pathway but with fragility of skills as they appear, or alternatively whether groups of children follow different developmental pathways.

\section{Methodological Implications}

In this study we introduced a new cross-notation comparison task in which children compared symbols with nonsymbolic representations of quantity. This task allowed very young children's mapping between representations to be explored. This task does not suffer from many of the limitations of existing dot comparison tasks (Inglis \& Gilmore, 2014; Price, Palmer, Battista, \& Ansari, 2012). Since each trial only includes a single dot array, problems with the visual characteristics of the arrays are reduced, and this reduces the inhibitory control demands of the task (Fuhs \& McNeil, 2013; Gilmore et al., 2013). Because the visual characteristics of the arrays do not need to be controlled in the same manner as for dot comparison tasks, a smaller number of trials is acceptable, making this task very suitable for use with young children. We found no evidence that children used counting to solve this task indicating that it is a good method to tap into the accuracy of children's mapping between symbolic and nonsymbolic representations.

We found that children's performance on a count list elicitation task gave only a limited picture of the importance of counting skills. Although there was a zero-order correlation between count list knowledge and mapping skills, this was fully accounted for by the relationship of both of these skills with cardinality understanding. Thus it was cardinality understanding, rather than simply count list knowledge, that appeared to play an important 
role in children's broader mathematical development. This adds to the weight of evidence that assessing count list knowledge provides only a limited picture of children's understanding of counting (e.g., Condry \& Spelke, 2008; Wynn, 1995) and that cardinality understanding is distinct from counting skill (Bermejo, Morales, \& deOsuna, 2004). In keeping with some previous research, our count elicitation task involved a set of objects for children to count (e.g., Le Corre \& Carey, 2007; Slaughter et al., 2006), while other studies have asked children to count without a set of objects (e.g., Barth et al., 2009; Lipton \& Spelke, 2005). The differences in the demands of these task versions have not been fully understood. It is thus important that researchers who are interested in the role of counting skills in the development of broader mathematical achievement should employ a set of tasks that capture the complex, multicomponential nature of counting skill.

\section{Implications for Supporting the Development of Early Number Skills}

Our findings show that there is a complex interplay between two distinct skills in young children's numeracy development. The first of these involves making connections between number words and quantities. Giving meaning to abstract numerical symbols is crucial for children's later success with mathematics (Mundy \& Gilmore, 2009) as it is the mapping between symbols and magnitudes that gives numbers their meaning. For some children at least, making the connection between numbers and magnitudes may actually help them come to understand the symbolic system itself, i.e. to understand cardinality. This suggests that activities which help children to link number words and quantities may be beneficial in promoting their understanding of the symbolic system. Activities such as guessing games, in which children estimate approximate numerosities, or board games (e.g., Siegler \& Ramani, 2008), in which they match symbols and magnitudes, may be ways to help children connect symbols and quantities. 
While some children appear to develop mapping skills first, our findings suggest that for other children, gaining sophisticated understanding of the symbolic counting system takes priority. Therefore, alongside activities to promote the connection between numbers and quantities, children should be given the opportunity to develop their understanding of the symbolic counting system itself. Simple activities that involve challenging children to reflect upon the number of items in a set have been found to successfully enhance children's understanding of cardinality (Bermejo et al., 2004). Engaging with a wide variety of activities involving counting may thus help children along the path to a rich understanding of numbers and all their meanings. 


\section{References}

Baroody, A. J., Wilkins, J. L. M., \& Tiilikainen, S. H. (2003). The development of children's understanding of additive commutativity: From protoquantitive concept to general concept? In A. J. Baroody \& A. Dowker (Eds.), The development of arithmetic concepts and skills: Constructing adaptive expertise (pp. 127 - 160). Mahwah, NJ: Erlbaum.

Barth, H., Kanwisher, N., \& Spelke, E. (2003). The construction of large number representations in adults. Cognition, 86, 201-221. doi: 10.1016/S0010-0277(02)00178-6

Barth, H., La Mont, K., Lipton, J., \& Spelke, E. S. (2005). Abstract number and arithmetic in preschool children. Proceedings of the National Academy of Sciences of the United States of America, 102, 14116-14121. doi: 10.1073/pnas.0505512102

Barth, H., Starr, A., \& Sullivan, J. (2009). Children's mappings of large number words to numerosities. Cognitive Development, 24, 248-264. doi: 10.1016/j.cogdev.2009.04.001

Bermejo, V., Morales, S., \& deOsuna, J. G. (2004). Supporting children's development of cardinality understanding. Learning and Instruction, 14, 381-398. doi: 10.1016/j.learninstruc.2004.06.010

Booth, J. L., \& Siegler, R. S. (2008). Numerical magnitude representations influence arithmetic learning. Child Development, 79, 1016-1031. doi: 10.1111/j.14678624.2008.01173.x

Brankaer, C., Ghesquière, P., \& De Smedt, B. (2014). Children's mapping between nonsymbolic and symbolic magnitudes and its association with timed and untimed tests of mathematics achievement. PLoS One, 9(4), e93565, 1-11. doi:

10.1371/journal.pone.0093565

Chen, Q. \& Li, J. (2014). Association between individual differences in non-symbolic number acuity and math performance: A meta-analysis. Acta Psychologica, 148, 163172. doi: 10.1016/j.actpsy.2014.01.016 
Condry, K. F., \& Spelke, E. S. (2008). The development of language and abstract concepts: the case of natural number. Journal of Experimental Psychology: General, 137(1), 22-38. doi: $10.1037 / 0096-3445.137 .1 .22$

Cowan R., Dowker, A., Christakis, A., \& Bailey, S. (1996). Even more precisely assessing children's understanding of the order-irrelevance principle. Journal of Experimental Child Psychology, 62, 84-101. doi: 10.1006/jecp.1996.0023

Dehaene, S., Dehaene-Lambertz, G., \& Cohen, L. (1998). Abstract representations of numbers in the animal and human brain. Trends in Neurosciences, 21, 355-361. doi: $10.1016 / \mathrm{S} 0166-2236(98) 01263-6$

Dehaene, S., Dupoux, E., \& Mehler, J. (1990). Is numerical comparison digital? Analogical and symbolic effects in two-digit number comparison. Journal of Experimental Psychology: Human Perception and Performance, 16, 626-641. doi: 10.1037/00961523.16.3.626

Dehaene, S., Spelke, E., Pinel, P., Stanescu, R., \& Tsivkin, S. (1999). Sources of mathematical thinking: Behavioral and brain-imaging evidence. Science, 284, 970-974. doi: $10.1126 /$ science. 284.5416 .970

De Smedt, B., Noël, M. P., Gilmore, C., \& Ansari, D. (2013). How do symbolic and nonsymbolic numerical magnitude processing skills relate to individual differences in children's mathematical skills? A review of evidence from brain and behavior. Trends in Neuroscience and Education, 2, 48-55. doi: 10.1016/j.tine.2013.06.001

Fazio, L. K., Bailey, D. H., Thompson, C. A., \& Siegler, R. S. (2014). Relations of different types of numerical magnitude representations to each other and to mathematics achievement. Journal of Experimental Child Psychology, 123, 53-72. doi: 10.1016/j.jecp.2014.01.013 
Feigenson, L., Dehaene, S., \& Spelke, E. (2004). Core systems of number. Trends in Cognitive Sciences, 8(7), 307-314. doi: 10.1016/j.tics.2004.05.002

Fuhs, M. W., \& McNeil, N. M. (2013). ANS acuity and mathematics ability in preschoolers from low-income homes: contributions of inhibitory control. Developmental Science, 16, 136-148. doi: 10.1111/desc.12013

Fuson, K. C., Pergament, G. G., Lyons, B. G., \& Hall, J. W. (1985). Children's conformity to the cardinality rule as a function of set size and counting accuracy. Child Development, 56, 1429-1436. doi: 10.2307/1130462

Gallistel, C. R., \& Gelman, R. (1992). Preverbal and verbal counting and computation. Cognition, 44, 43-74. doi: 10.1016/0010-0277(92)90050-R

Gelman, R., \& Gallistel, C. R. (1978). The child's understanding of number. Cambridge, MA: Harvard University Press.

Gilmore, C., Attridge, N., Clayton, S., Cragg, L., Johnson, S., Marlow, N., Simms, V., \& Inglis, M. (2013). Individual differences in inhibitory control, not non-verbal number acuity, correlate with mathematics achievement. PloS one, 8, e67374. doi: 10.1371/journal.pone.0067374

Gilmore, C. K., McCarthy, S. E., \& Spelke, E. S. (2007). Symbolic arithmetic knowledge without instruction. Nature, 447, 589-591. doi:10.1038/nature05850

Gilmore, C. K., \& Papadatou-Pastou, M. (2009). Patterns of individual differences in conceptual understanding and arithmetical skill: A meta-analysis. Mathematical Thinking and Learning, 11, 25-40. doi:10.1080/10986060802583923

Huntley-Fenner, G., \& Cannon, E. (2000). Preschoolers' magnitude comparisons are mediated by a preverbal analog mechanism. Psychological Science, 11, 147-152. doi: $10.1111 / 1467-9280.00230$ 
Inglis, M., \& Gilmore, C. (2014). Indexing the approximate number system. Acta Psychologica, 145, 147-155. doi: 10.1016/j.actpsy.2013.11.009

Izard, V., \& Dehaene, S. (2008). Calibrating the mental number line. Cognition, 106, 12211247. doi: 10.1016/j.cognition.2007.06.004

Le Corre, M., \& Carey, S. (2007). One, two, three, four, nothing more: An investigation of the conceptual sources of the verbal counting principles. Cognition, 105, 395-438. doi: 10.1016/j.cognition.2006.10.005

Lipton, J. S., \& Spelke, E. S. (2005). Preschool children's mapping of number words to nonsymbolic numerosities. Child Development, 76, 978-988. doi: 10.1111/j.14678624.2005.00891.x

McCrink, K., \& Wynn, K. (2004). Large-number addition and subtraction by 9-month-old infants. Psychological Science, 15, 776-781. doi: 10.1111/j.0956-7976.2004.00755.x

Moyer, R. S., \& Landauer, T. K. (1967). Time required for judgements of numerical inequality. Nature, 215, 1519-1520. doi:10.1038/2151519a0

Mundy, E., \& Gilmore, C. K. (2009). Children's mapping between symbolic and nonsymbolic representations of number. Journal of Experimental Child Psychology, 103, 490-502. doi: 10.1016/j.jecp.2009.02.003

Mussolin, C., Nys, J., \& Leybaert, J. (2012). Relationships between approximate number system acuity and early symbolic number abilities. Trends in Neuroscience and Education, 1(1), 21-31. doi: 10.1016/j.tine.2012.09.003

Price, G. R., Palmer, D., Battista, C., \& Ansari, D. (2012). Nonsymbolic numerical magnitude comparison: reliability and validity of different task variants and outcome measures, and their relationship to arithmetic achievement in adults. Acta Psychologica, 140(1), 50-57. doi: 10.1016/j.actpsy.2012.02.008 
Sarnecka, B. W., \& Gelman, S. A. (2004). Six does not just mean a lot: preschoolers see number words as specific. Cognition, 92, 329-352. doi: 10.1016/j.cognition.2003.10.001

Siegler, R. S., \& Crowley, K. (1991). The microgenetic method: A direct means for studying cognitive development. American Psychologist, 46, 606-620. doi: 10.1037/0003066X.46.6.606

Siegler, R. S., \& Ramani, G. B. (2008). Playing linear numerical board games promotes lowincome children's numerical development. Developmental Science, 11, 655-661. doi: $10.1111 / \mathrm{j} .1467-7687.2008 .00714 . x$

Slaughter, V., Kamppi, D., \& Paynter, J. (2006). Toddler subtraction with large sets: further evidence for an analog-magnitude representation of number. Developmental Science, 9(1), 33-39. doi: 10.1111/j.1467-7687.2005.00460.x

Slusser, E. B., \& Sarnecka, B. W. (2011). Find the picture of eight turtles: A link between children's counting and their knowledge of number word semantics. Journal of Experimental Child Psychology, 110(1), 38-51. doi: 10.1016/j.jecp.2011.03.006

Temple, E., \& Posner, M. I. (1998). Brain mechanisms of quantity are similar in 5-year-old children and adults. Proceedings of the National Academy of Sciences, 95, 7836-7841. doi: $10.1073 /$ pnas.95.13.7836

Wagner, J. B., \& Johnson, S. C. (2011). An association between understanding cardinality and analog magnitude representations in preschoolers. Cognition, 119(1), 10-22. doi: 10.1016/j.cognition.2010.11.014

Wood, J. N., \& Spelke, E. S. (2005). Infants' enumeration of actions: numerical discrimination and its signature limits. Developmental Science, 8(2), 173-181. doi: $10.1111 / \mathrm{j} .1467-7687.2005 .00404 . x$

Wynn, K. (1990). Children's understanding of counting. Cognition, 36(2), 155-193. doi: $10.1016 / 0010-0277(90) 90003-3$ 
Wynn, K. (1992). Children's acquisition of the number words and the counting system. Cognitive Psychology, 24, 220-251. doi: 10.1016/0010-0285(92)90008-P

Wynn, K. (1995). Origins of numerical knowledge. Mathematical Cognition, 1, 35-60

Wynn, K. (1996). Infants' individuation and enumeration of actions. Psychological Science, 7(3), 164-169. doi: 10.1111/j.1467-9280.1996.tb00350.x

Wynn, K., Bloom, P., \& Chiang, W. C. (2002). Enumeration of collective entities by 5month-old infants. Cognition, 83, B55-B62. doi: 10.1016/S0010-0277(02)00008-2

Xenidou-Dervou, I, De Smedt, B, van der Schoot, M., \& van Lieshout, E. C. D. M. (2013). Individual differences in kindergarten math achievement: The integrative roles of approximation skills and working memory. Learning and Individual Differences, 28 , 119-129. doi: 10.1016/j.lindif.2013.09.012 


\section{Footnotes}

1. Note that in the case of small numbers (less than 4), success may depend on set size rather than ratio. There is ongoing debate as to whether small numbers engage the same approximate number system as larger numbers (see Feigenson, Dehaene \& Spelke, 2004, for an overview of the two core systems hypothesis).

2. These results are replicated when using non-parametric statistics. 
Table 1

Cross-Notation Comparison Problems

\begin{tabular}{|c|c|c|c|}
\hline Problem Number & Distance & $\begin{array}{l}\text { Quantity } 1 \\
\text { (dot array) }\end{array}$ & $\begin{array}{l}\text { Quantity } 2 \\
\text { (verbal number word) }\end{array}$ \\
\hline 1 & Small & 1 & 3 \\
\hline 2 & Small & 3 & 2 \\
\hline 3 & Large & 1 & 5 \\
\hline 4 & Large & 6 & 2 \\
\hline 5 & Small & 5 & 4 \\
\hline 6 & Small & 7 & 5 \\
\hline 7 & Large & 4 & 8 \\
\hline 8 & Large & 5 & 10 \\
\hline 9 & Small & 6 & 9 \\
\hline 10 & Small & 7 & 10 \\
\hline 11 & Large & 9 & 4 \\
\hline 12 & Large & 10 & 6 \\
\hline
\end{tabular}

Note. Quantity 1 indicates the number of dots presented on the card and Quantity 2 indicates the verbal number word given. 
Table 2

Number of Children at Each Knower-Level According to Performance on the Give-a-Number Task and Mean Age of Children in Each Group

\begin{tabular}{|c|c|c|}
\hline Knower level & Number of children & Mean age \\
\hline 0 & 2 & 36.5 \\
\hline 1 & 8 & 38.1 \\
\hline 2 & 10 & 38.0 \\
\hline 3 & 4 & 40.0 \\
\hline 4 & 5 & 48.2 \\
\hline Cardinal Principle & 23 & 51.0 \\
\hline
\end{tabular}


Figure 1 Experimental Set-up of the Cross-Notation Comparison Task.

a)

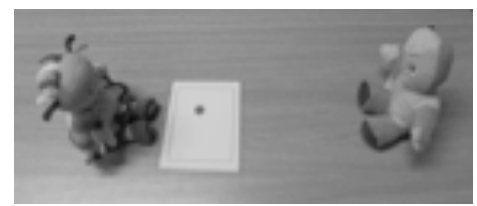

"Upsy Daisy has this many

balls"

b)

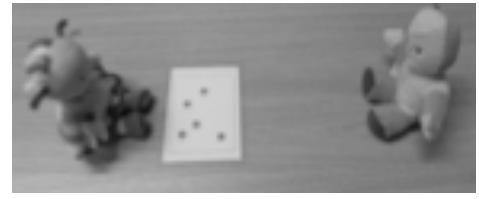

"Upsy Daisy has this many balls"

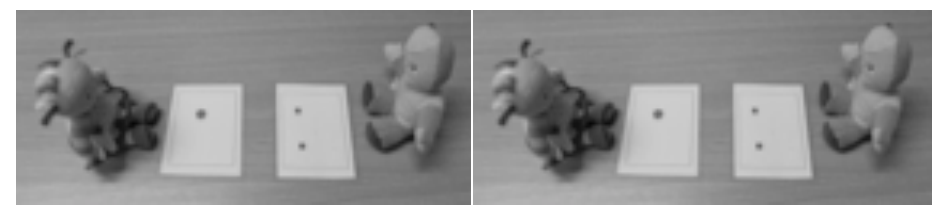

"Iggle Piggle has this

"Who has the most balls?"

many balls"

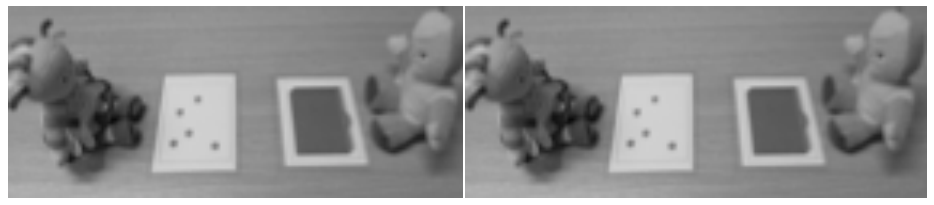

"Iggle Piggle has hidden "Who has the most balls?" his balls in a box. Iggle

Piggle has ten balls"

Figure1: Part a) gives an example of a nonsymbolic (dot comparison) practice trial and part b) gives an example of a cross-notation comparison trial. 
Figure 2 Scatterplots Depicting the Relationships Among Performance on the Three Tasks
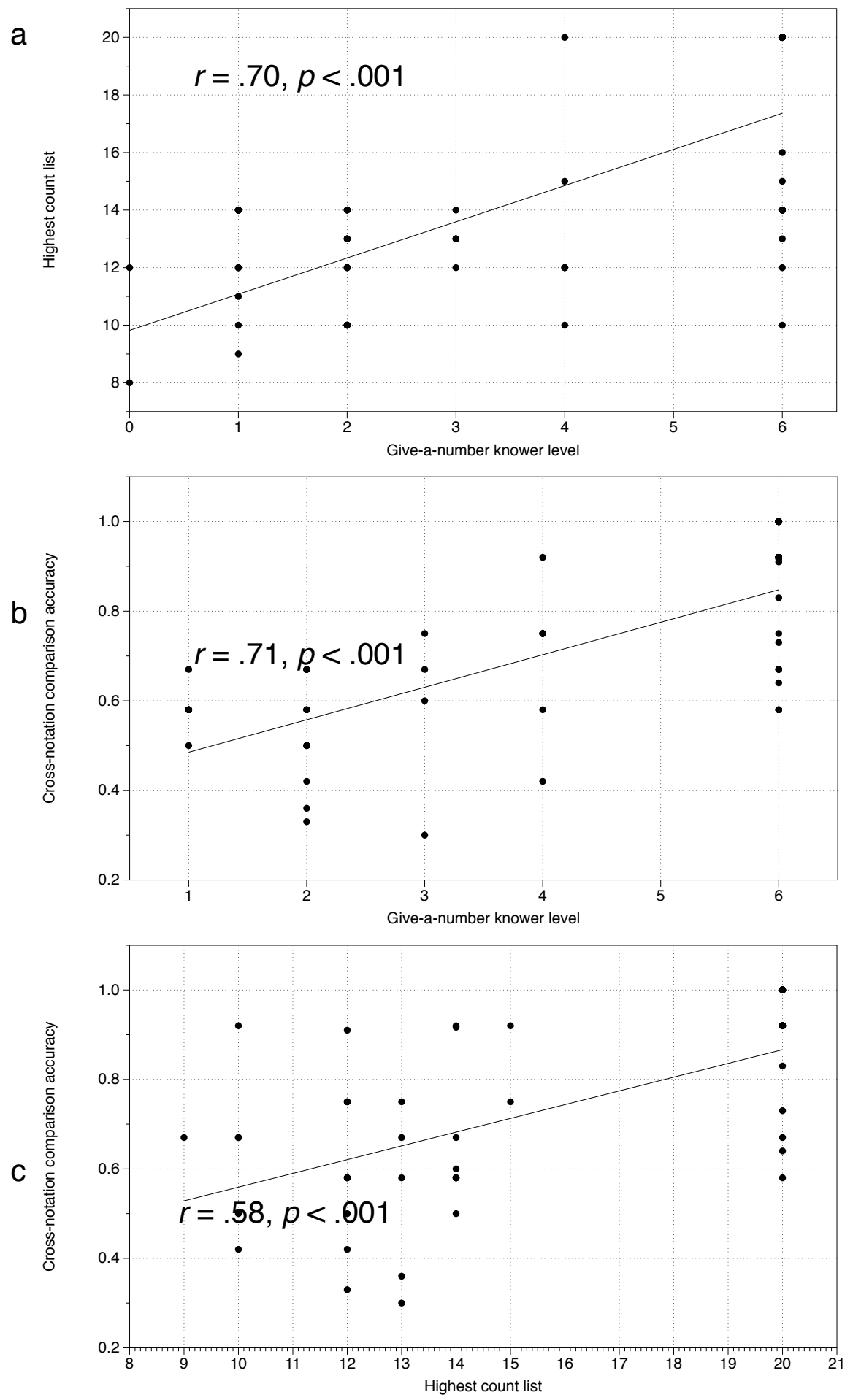

Figure 2: a) Give-a-Number task and count list elicitation b) Give-a-Number task and cross-notation comparison task and c) cross-notation comparison task and count list elicitation. 

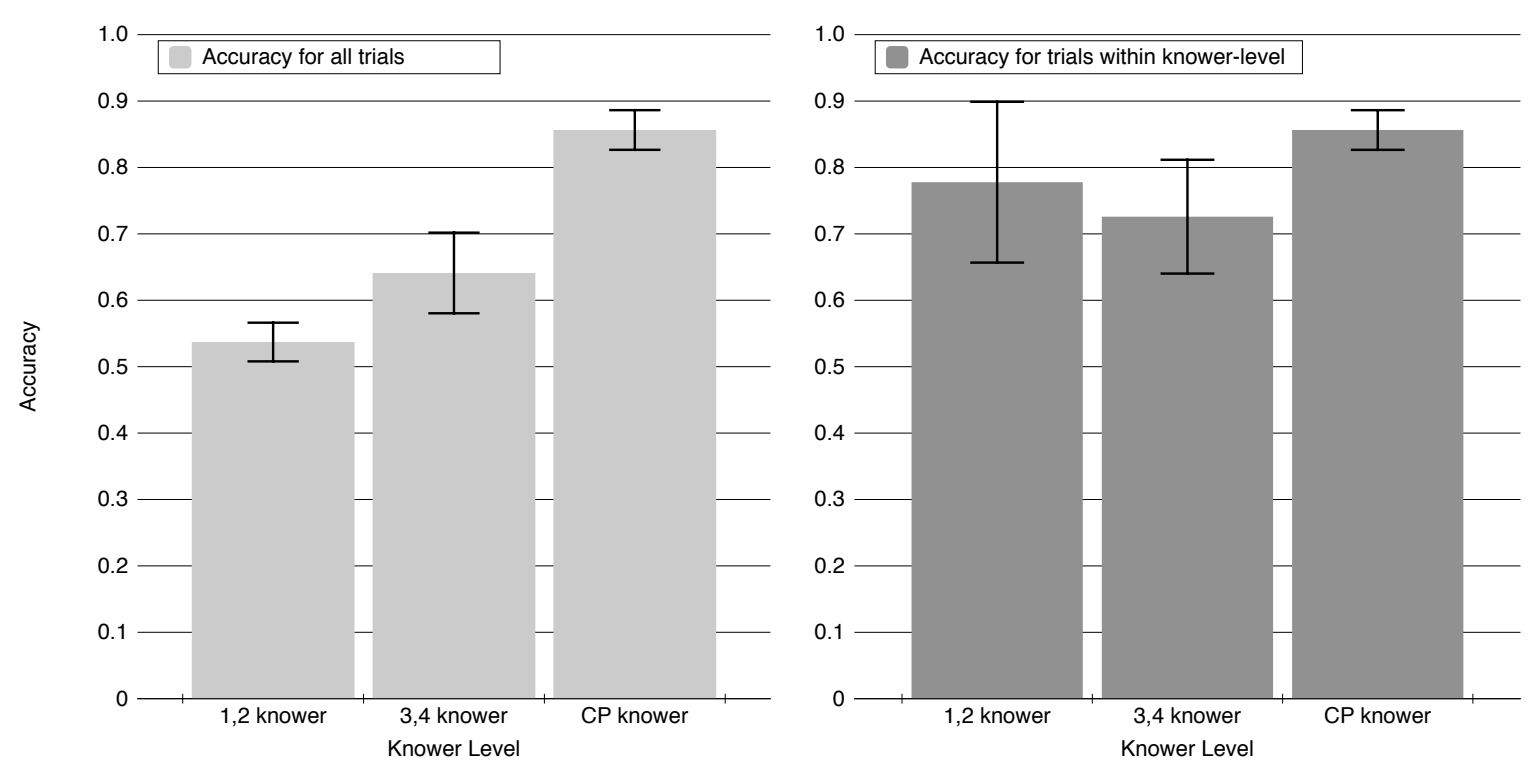

Figure 3. Performance on the cross-notation comparison task according to knower-level showing accuracy on the full set of trials and the subset of trials which fall within knowerlevel range. 